\title{
Novel visible-range luminescence of pristine nanozirconia phosphor using green fabrication techniques
}

\author{
G DEEPTHI REDDY ${ }^{1, *} \mathbb{1}$, M NOORJAHAN $^{1}$ and A RATNAMALA ${ }^{2}$ \\ ${ }^{1}$ Department of Chemistry, Palamuru University, Mahbubnagar 509001, India \\ ${ }^{2}$ Department of Chemistry, GITAM University, Bengaluru Campus, Bangalore 560065, India \\ *Author for correspondence (deepalirreddy@ gmail.com)
}

MS received 27 March 2018; accepted 9 July 2018; published online 2 February 2019

\begin{abstract}
We report unification of pristine nanozirconia phosphor (PNZP), which exhibits orange-red luminescence. PNZP was developed in a lucid manner by the reduction of a zirconyl oxy chloride $\left(\mathrm{ZrOCl}_{2} \cdot 8 \mathrm{H}_{2} \mathrm{O}\right)$ solution with a wet leaf extract of Syzygium cumini (S. cumini) using green fabrication techniques (ultrasonication and microwave irradiation) in the absence of a surfactant and a base, which opens a new avenue for designing novel visible-range luminescence nanophosphors. The ultraviolet-visible spectrum of the aqueous medium containing zirconia nanoparticles showed an absorption peak at around $262 \mathrm{~nm}$. Powder X-ray diffraction study showed that the particles are crystalline in nature, with an average size of $\sim 2.06 \mathrm{~nm}$ comprising a tetragonal/monoclinic $\mathrm{ZrO}_{2}$ structure. Photoluminescence spectroscopy study has shown a sharp emission peak at $603 \mathrm{~nm}$ and broad emission peaks at 670 and $720 \mathrm{~nm}$ at $350 \mathrm{~nm}$ excitation. The most useful outcome of this work will be the development of pure nanophosphors using plant extracts which contain different fluorophores, with applications in nanoelectronic devices, catalysis, optoelectronics and piezoelectric devices.
\end{abstract}

Keywords. Pristine nanozirconia; phosphor; luminescence; green fabrication.

\section{Introduction}

In the past decades zirconium oxide has attracted great attention because of its versatile applications in diversified areas such as a catalyst support, as an insulator in transistors for nanoelectronic devices, in solid oxide fuel cells, optoelectronics, piezoelectric and nanocomposite devices [1-4]. The mechanical properties of zirconia are well acknowledged in the literature because of its superior characteristics like good thermal expansion coefficient, stability of phase, high fracture toughness etc. $[5,6]$. The nanophosphors are also well studied because of their high refractive index and ability to absorb high-energy radiation. More attention has been focused on nanocrystalline nanophosphors because of the increased size-dependent beneficial properties which enhance the luminescence characteristics [7] for applications in lighting devices. Also, the materials with short wavelength light-emitting properties are of most interest due to higher information storage capacity in compact discs [8].

Phosphors may exist in the form of nanoparticles, nanorods, nanowires, nanotubes as well as colloidal or bulk nanocrystals. The luminescence of nanophosphor materials usually comes from the band gap and the various defects in which the band gap and defect emission of phosphor materials might be located in the ultraviolet (UV) and visible (400$600 \mathrm{~nm}$ ) regions. The synthesis of nanophosphors is generally done by physico-chemical methods such as using an ionized cluster beam, chemical precipitation, sol-gel technique, auto-combustion, co-precipitation etc. [9]. All these methods were employed to synthesize a material in order to exhibit unique physical and chemical properties to achieve a limited size and a high density of corner or edge surface sites. However, green synthesis is an alternative option to the abovementioned methods to synthesize nanomaterials exhibiting nanophosphor properties as these methods are simple, rapid and environmentally benign. Literature based on this energy upholding process is limited [10].

Majedi et al reported the synthesis of cubic phase zirconia nanoparticles using a mixture of lemon juice and sucrose as a precursor [11] and Gurushantha et al reported the synthesis of zirconia nanocomposites using Phyllanthus acidus as a precursor [12]. Herein, we report a green fabricated pristine nanozirconia phosphor (PNZP) material emitting orangered luminescence developed by the reduction of a zirconyl oxy chloride $\left(\mathrm{ZrOCl}_{2} \cdot 8 \mathrm{H}_{2} \mathrm{O}\right)$ solution with Syzygium cumini (S. cumini) wet leaf extract without using any surfactant and base through green techniques such as microwave irradiation and ultrasonication, which opens a new avenue for designing visible range luminescence nanophosphors. This work presents a general low-cost, eco-friendly approach for designing PNZP material of quantum size exhibiting visible range luminescence through single-step green techniques. 


\section{Experimental}

\subsection{Materials}

Zirconyl oxy chloride $\left(\mathrm{ZrOCl}_{2} \cdot 8 \mathrm{H}_{2} \mathrm{O} 97 \%\right)$ was purchased from Fluka AG, Buchs SG, and used without further purification. S. cumini leaves were collected from local sources. All aqueous solutions were prepared using distilled water (DW).

\subsection{Synthesis of PNZP particles}

S. cumini leaves $(100 \mathrm{~g})$ were collected and washed several times with DW to eliminate foreign particles and the leaves were ground using a pestle and mortar. The mixture was placed in a beaker with DW, stirred for half an hour to obtain uniform distribution throughout the medium and then the solution was filtered using Whatman filter paper to get a pure extract of $S$. cumini. This freshly prepared supernatant was transferred into beakers labelled $\mathrm{A}\left(Z_{\mathrm{MW}}\right), \mathrm{B}\left(Z_{\mathrm{US}}\right)$ and $\mathrm{C}\left(Z_{\mathrm{RT}}\right)$. Each beaker was filled with $100 \mathrm{ml}$ supernatant and $0.01 \mathrm{M} \mathrm{ZrOCl}_{2} \cdot 8 \mathrm{H}_{2} \mathrm{O}$ solution was added. An immediate colour change was observed in the beakers. The two solutions in beakers A and B were subjected to microwave irradiation and ultrasonication and the solution in beaker $\mathrm{C}$ was kept undisturbed at room temperature (RT) for $24 \mathrm{~h}$.

Reaction mixtures labelled with $\mathrm{A}\left(Z_{\mathrm{MW}}\right)$ and $\mathrm{B}\left(Z_{\mathrm{US}}\right)$ were stirred vigorously for $1 \mathrm{~h}$ at RT and then subjected to microwave irradiation $\left(Z_{\mathrm{MW}}\right)$ for $4 \mathrm{~min}$ at $800 \mathrm{~W}$ and to ultrasonication $\left(Z_{\mathrm{US}}\right)$ treatment for $15 \mathrm{~min}$, respectively. The reaction mixtures $\mathrm{A}\left(Z_{\mathrm{MW}}\right)$ and $\mathrm{B}\left(Z_{\mathrm{US}}\right)$ were allowed to stand for $24 \mathrm{~h}$ at RT and then washed with DW and with ethanol in order to remove impurities and dried in an oven at $100^{\circ} \mathrm{C}$. The dried samples of $Z_{\mathrm{US}}, Z_{\mathrm{MW}}, Z_{\mathrm{RT}}$ were subjected to a calcination procedure in a muffle furnace at $500^{\circ} \mathrm{C}$ for $5 \mathrm{~h}$ for the formation of the PNZP particles. The nanoparticles obtained by dehydration of the as-prepared sample resulted in a white powder of PNZP. Figure 1 shows a flow chart of the synthesis procedure of PNZP particles.

\subsection{Instrumentation}

X-ray diffraction (XRD) studies of PNZP were carried out on an X'pert Pro X-ray diffractometer (Panalytical B.V., the Netherlands) using Ni-filtered $\mathrm{Cu} \mathrm{K} \alpha$ radiation (=1.5406) from $2 \theta=0-60$ operating at $40 \mathrm{kV}$ and a current of $30 \mathrm{~mA}$ at a scan rate of $0.388 \mathrm{~min}^{-1}$ to determine the crystalline phase and structure. The morphology of PNZPs was observed using scanning electron microscopy (SEM) of ZEISS EVO 18 model. Images of the PNZPs were obtained using transmission electron microscopy (TEM) of JEOL JSM-6500F model with an accelerating voltage of $200 \mathrm{kV}$ to observe the morphology. The specimen for TEM imaging was prepared from the particles suspension in isopropanol. A drop of well-dispersed supernatant was placed on a carbon-coated 200 mesh copper grid, followed by drying the sample under ambient conditions before it was attached to the material holder on the microscope. The UV-vis spectra of the samples were recorded with a Shimadzu-1800 spectrophotometer in the range of 200-1100 nm. Photoluminescence of the samples was recorded using a Molecular Devices (SpectraMax M3) UV-visible spectrophotometer operating at a resolution of $1 \mathrm{~nm}$.

\section{Results}

\subsection{XRD analysis}

The XRD patterns of the PNZP particles calcined at $500^{\circ} \mathrm{C}$ is recorded to determine the phase formation of nanophosphors and the results are shown in figure 2. XRD patterns clearly show the formation of highly crystalline $\mathrm{ZrO}_{2}$ due to its sharp and narrow line widths. The diffraction patterns of PNZP powder shows a tetragonal phase at $2 \theta 30.22^{\circ}, 34.9^{\circ}$, $50.34^{\circ}$ and $60.1^{\circ}$, which correspond to (101), (110), (200) and (211) and a characteristic diffraction pattern [JCPDS No. 791771] at $2 \theta$ of $74.14^{\circ}$, which corresponds to a monoclinic phase. All the results are well in accordance with the literature reports $[13,14]$. The average crystallite sizes of the $\mathrm{ZrO}_{2}$ nanocrystallites have been estimated by Scherer's formula. The approximate size of PNZP particles was found to be $2.06 \mathrm{~nm}$, which indicates that calcination of the samples at $500^{\circ} \mathrm{C}$ for $5 \mathrm{~h}$ results in small nuclei of zirconium crystals without much agglomeration of particles in the mixture.

\subsection{SEM and TEM}

Surface morphology and chemical composition of the $\mathrm{ZrO}_{2}$ nanostructures were analysed by using a SEM equipped with energy-dispersive X-ray spectroscope as shown in figure 3A. The present study shows that zirconium oxide is observed as perfect dispersed spherical balls and rods under SEM analysis $[14,15]$. The structures are of irregular morphology, may be due to a slight agglomeration of particles. EDAX analysis shows signal characteristics of $\mathrm{Zr}$ and $\mathrm{O}$ without any impurities.

Figure 3B, shows the TEM images of PNZP prepared using microwave irradiation and ultrasonication techniques with a uniform size and well-defined morphology. As can be seen from TEM images, there is slight or very little agglomeration with a mean crystallite size range of nearly $2-6 \mathrm{~nm}$. It is noteworthy that a well-controlled uniform nanoparticle size with good morphology has been achieved through ultrasonic wave and microwave irradiation without using any surfactant and base through green techniques.

\subsection{UV-vis spectra}

UV-vis absorption spectrum was recorded for PNZP material synthesized by microwave irradiation and ultrasonication in the wavelength range $200-800 \mathrm{~nm}$ as shown in figure 4 . 


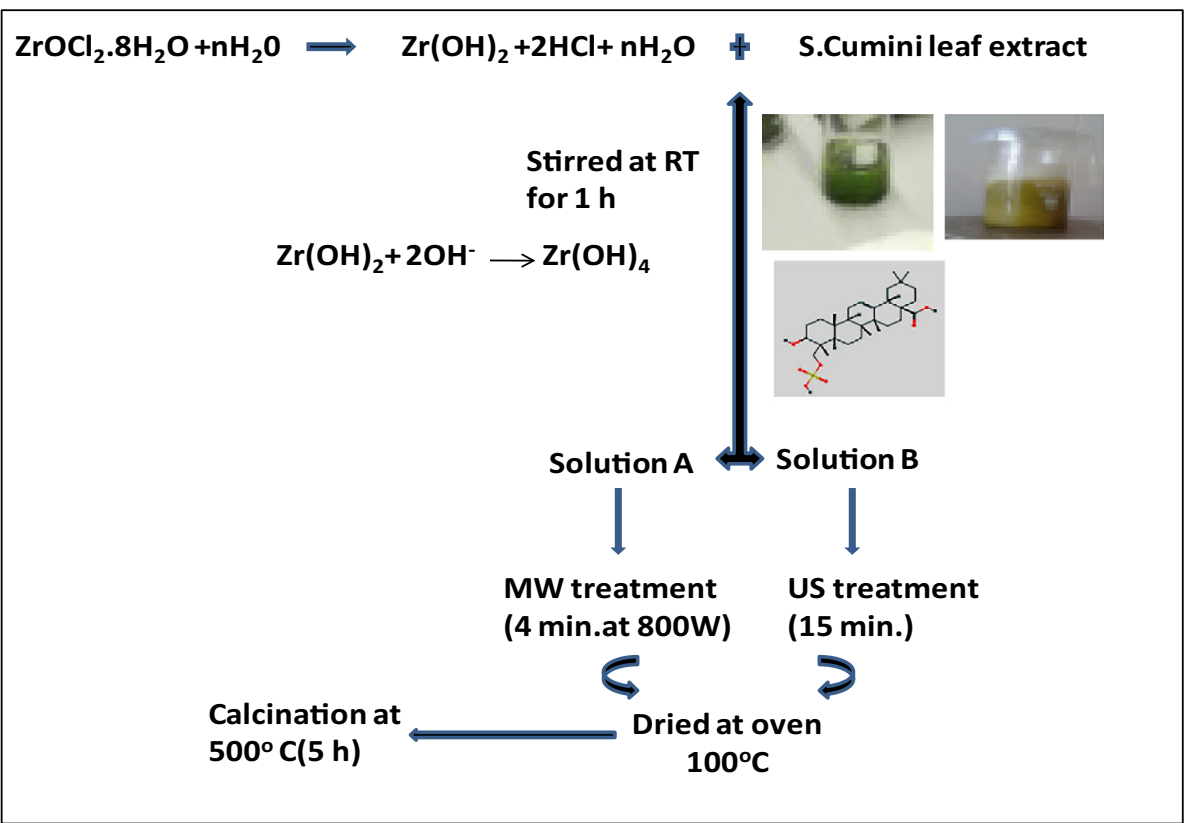

Figure 1. Schematic of green fabrication techniques for the unification of PNZP particles using S. cumini leaf extract.

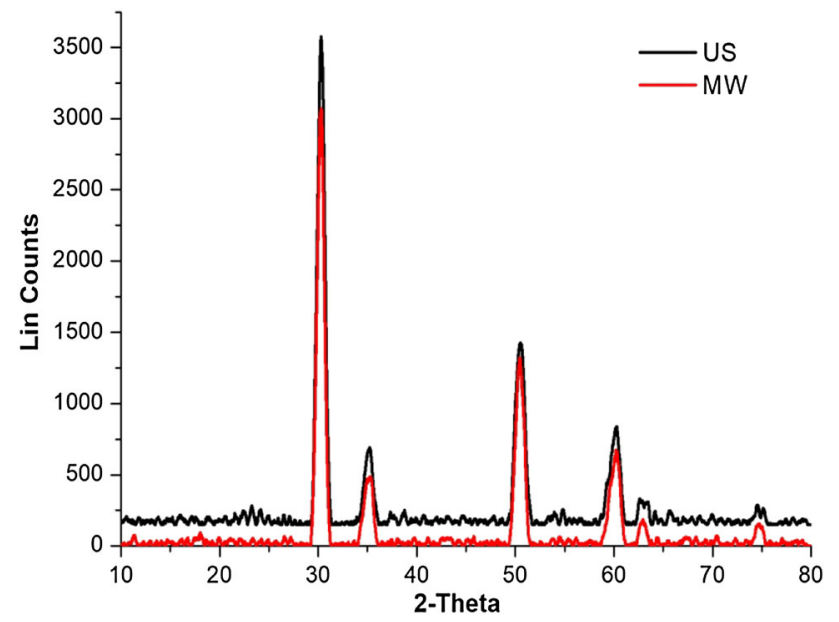

Figure 2. XRD patterns of ultrasonicated $\left(Z_{\mathrm{US}}\right)$ and microwave irradiated $\left(Z_{\mathrm{MW}}\right)$ PNZP particles.

The spectrum shows a sharp and prominent absorption maxima at $\sim 215-235 \mathrm{~nm}(5.78-5.28 \mathrm{eV})$, which is due to the excitation of electrons or due to the $\mathrm{d}$ transition of $\mathrm{Zr}^{4+}$ and other weak absorption maxima are observed at $\sim 258-290 \mathrm{~nm}$ $(4.81-4.28 \mathrm{eV})$ and $\sim 640-650 \mathrm{~nm}(1.94-1.91 \mathrm{eV})$ may be because of transition involving extrinsic states such as defects and surface trap sites [15]. The optical band gaps determined for PNZP material from the absorption spectra are about 5.1$4.6 \mathrm{eV}$ and $4.13-3.60 \mathrm{eV}$.

\subsection{Photoluminescence spectra}

As such the $\mathrm{ZrO}_{2}$ does not show any luminescence unless there are impurities or dopants, phase transformation, and structural defects which incorporate the electronic states in the band gap. When PNZP materials are excited at $\lambda_{\max }=$ $350 \mathrm{~nm}$, a sharp orange-red photoluminescence is observed at $\sim 603 \mathrm{~nm}(2.0 \mathrm{eV})$ and broad emission at $\sim 670 \mathrm{~nm}$ $(1.8 \mathrm{eV})$ and $\sim 720 \mathrm{~nm}(1.72 \mathrm{eV})$ for both microwave irradiation $\left(Z_{\mathrm{MW}}\right)$ and ultrasonication $\left(Z_{\mathrm{US}}\right)$ treated samples. $Z_{\mathrm{RT}} \mathrm{RT}$ synthesis of PNZP particles shows a violet-blue luminescence at $\sim 480 \mathrm{~nm}(2.58 \mathrm{eV})[16]$ as shown in figure 5. Pure $S$. cumini leaf extract PL (inserted graph) is shown as a control measurement in figure 5. Liang et al also reported the red-shifted PL of microwave irradiated tetragonal zirconia nanoparticles excited at $412 \mathrm{~nm}$ [17]. The high intensity and red shift of PL is accredited to the existence of deep-level defect states and quantum confinement of electron-hole pairs. The existence of defect states that is in tune with UV-visible absorption is confirmed by the significant decrease in the optical band gaps due to the presence of oxygen vacancies/interstitials in PNZP material, which induces the formation of new energy levels in the band gap region [15]. The complete visible emission at $\sim 603 \mathrm{~nm}(2.0$ $\mathrm{eV}), \sim 670 \mathrm{~nm}(1.8 \mathrm{eV})$ and $\sim 720 \mathrm{~nm}(1.72 \mathrm{eV})$ is accredited to the singly ionized oxygen vacancies in PNZP materials, where the emission results from the radiative recombination of photogenerated holes with an electron occupying the oxygen vacancy, which confirms the quantum confinement model. In the present photoluminescence study of PNZP 
(A)

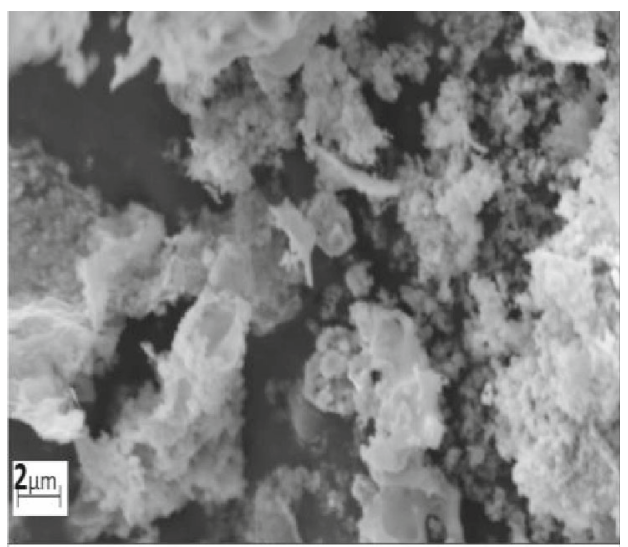

(Zus)

(B)

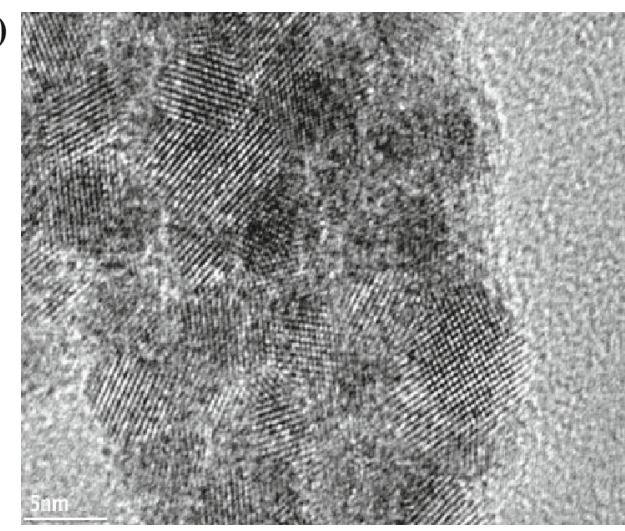

( $\left.\mathrm{Z}_{\mathrm{US}}\right)$

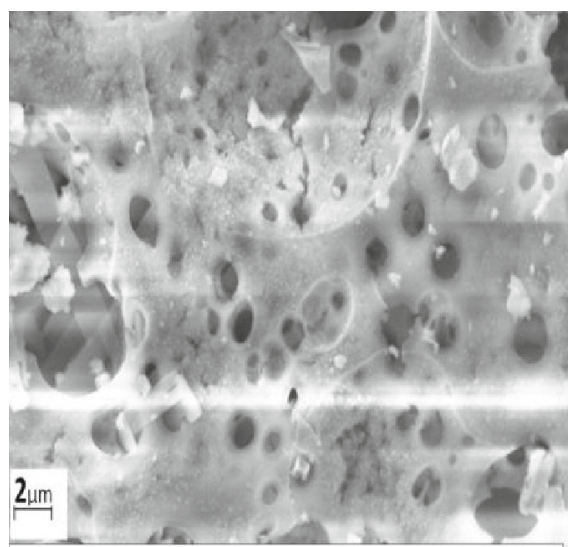

(ZMW)

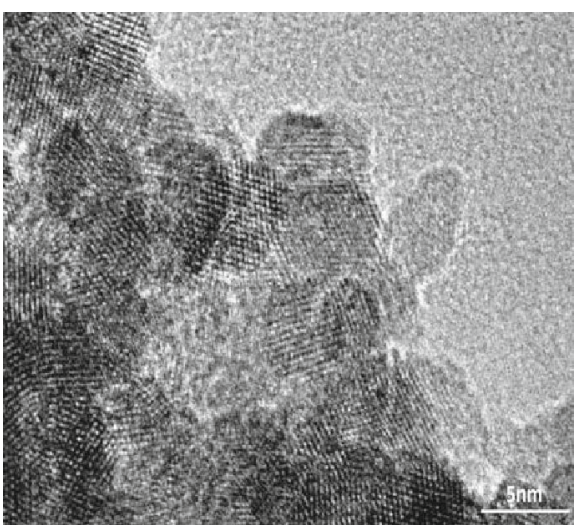

( $\left.\mathrm{Z}_{\mathrm{MW}}\right)$

Figure 3. (A) SEM images of ultrasonicated $\left(Z_{U S}\right)$ and microwave irradiated $\left(Z_{M W}\right)$ PNZP particles showing the surface morphology at $2 \mu \mathrm{m}$. (B) TEM micrographs of ultrasonicated ( $\left.Z_{\mathrm{US}}\right)$ and microwave irradiated $\left(Z_{\mathrm{MW}}\right)$ PNZP particles.

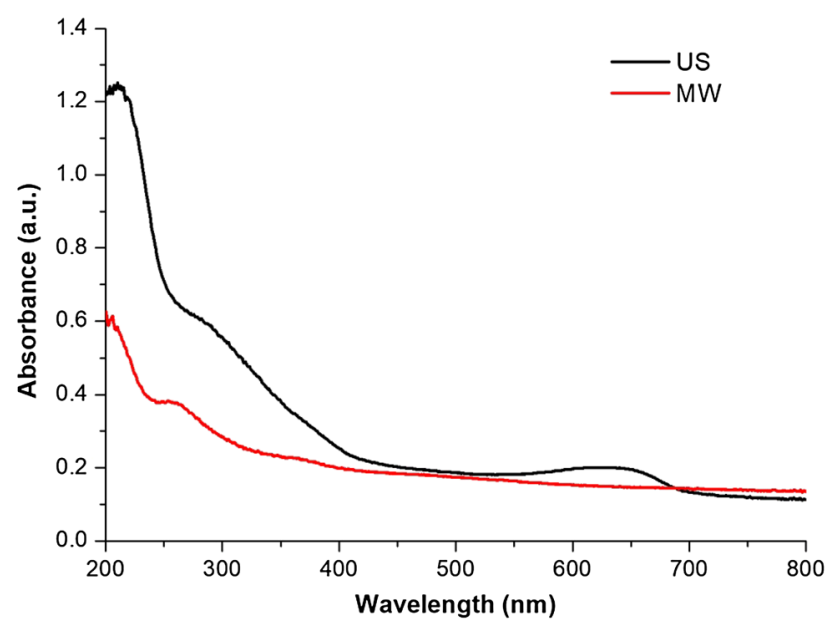

Figure 4. UV-visible absorption spectra of ultrasonicated $\left(Z_{\mathrm{US}}\right)$ and microwave irradiated $\left(Z_{\mathrm{MW}}\right)$ PNZP particles. material, there is no non-radioactive defect even though the average diameter of the particle is $\sim 2.06 \mathrm{~nm}$ because the surface of PNZP material is not well passivated that supports any kind of unrigorous bonds which would quench the radiative transitions $[18,19]$.

\subsection{Mechanism of formation of PNZP}

The mechanism of formation of nanophosphors is according to the starting materials of the chemical reaction. Zirconium hydroxide is formed in the reaction mixture with some byproducts due to the presence of acetyl flavanol glycosides, triterpenoids present in the $S$. cumini leaf extract $[20,21]$. The microwave and ultrasonic wave irradiation aids in hydrolysis, promotes the formation of $\mathrm{OH}^{\bullet}$ free radicals by decomposition of water molecules. When the zirconium hydroxide precipitate is subjected to microwave irradiation in beaker $Z_{\mathrm{MW}}$ and ultrasonic wave in beaker $Z_{\mathrm{US}}$, hot spots are formed which induces the formation of hydrous zirconia 


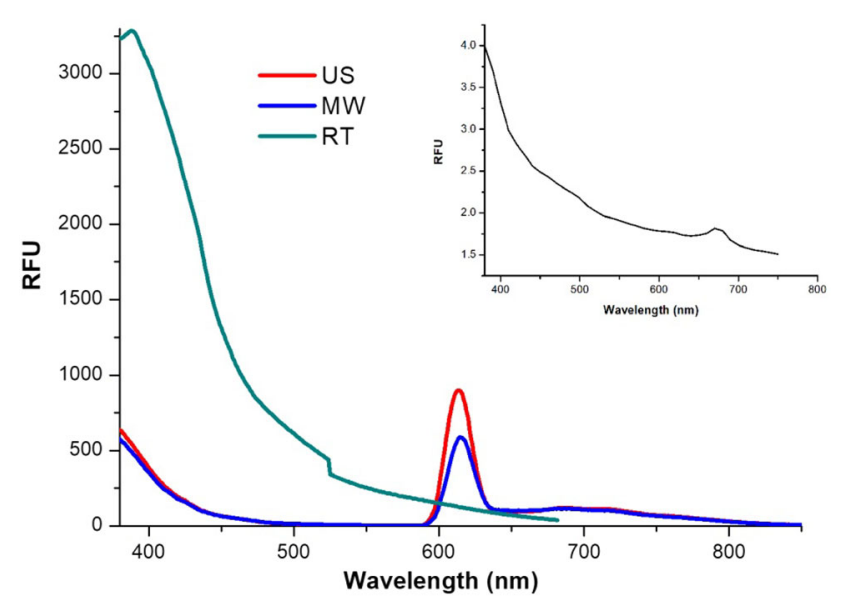

Figure 5. Photoluminescence spectra for ultrasonicated $\left(Z_{\mathrm{US}}\right)$, microwave irradiated $\left(Z_{\mathrm{MW}}\right)$, RT $\left(Z_{\mathrm{RT}}\right)$ PNZP particles and pure $S$. cumini leaves extract (inserted graph).

nanoparticles in the solution. On further washing with DW and ethanol and drying, PNZP particles are obtained.

\section{Conclusion}

Herein, we report a rapid and ecofriendly route for producing a neoteric and economical PNZP material showing visible range luminescence. Further research is in progress to examine the advantages of PNZP materials in catalytic, optoelectronic and bio-medical applications.

\section{Acknowledgements}

The authors thank Professor K Girija Mangatayaru, Head of Department of Chemistry, Palamuru University for her incredible support and acknowledge the DST-INSPIRE (AORC) Fellowship, New Delhi.

\section{References}

[1] Kitai A 2008 Luminescent materials and applications (New York, NY: John Wiley \& Sons) ISBN: 978-0-470-05818-3

[2] Pandey A, Dutta S, Kumar A, Raman R, Kapoor A K and Muralidhran R 2016 Adv. Mater. Lett. 7777

[3] Iorgu A, Berger D, Alexandrescu L, Vasile B S and Matei C 2013 Chalcogenide Lett. 10525

[4] Reshma, Sandupatla R and Veerasomaiah P 2016 Int. J. Nanomater. Biostruct. 630

[5] Anh T K, Benalloul P C, Barthou C, Giang L T K, Vu N and Minh L Q 2007 J. Nanomater. 2007 Article ID 48247

[6] Harish C 2006 Proc. ASID 8-12 Oct, New Delhi

[7] Balaji S, Mandal B K, Ranjan S, Dasgupta N and Chidambaram R 2017 J. Photochem. Photobiol.: B 170125

[8] Anandan K and Rajendran V 2013 J. Phys. Sci. 7179

[9] Liu X, Lu G and Yan Z 2003 J. Nat. Gas Chem. 12161

[10] Xu X and Wang X 2009 Nano. Res. 2891

[11] Majedi A, Abbasi A and Davar F 2016 J. Sol. Gel. Sci. Technol. 77542

[12] Gurushantha K, Anantharaju K S, Nagabhushana H, Sharma S C, Vidya Y S, Shivakumara C et al 2015 J. Mol. Catal. 397 36

[13] Zhao J, Fan W, Wu D and Sun Y 2000 J. Non-Crystal. Solids 26115

[14] Banerjee K, Prithviraj M, Augustine N, Pradeep S P and Thiagarajan P 2016 J. Chem. Pharm. Sci. 91186

[15] Kumari L, Du G H, Li W Z, Vennila R S, Saxena S K and Wang D Z 2009 Ceram. Int. 352401

[16] Siripireddy B, Badal Kumar M, Shivendu R, Nandita D G and Ramalingam C 2017 J. Photochem. Photobiol. B: Biol. 170 125

[17] Liang J, Deng Z, Jiang X, Li F and Li Y 2002 Inorg. Chem. 41 3602

[18] Ledoux G, Gong J, Huisken F, Guillois O and Reynaud Reynaud C 2002 Appl. Phys. Lett. 804834

[19] Liqiang J, Yichun Q, Baiqi W, Shudan L, Baojiang J, Libin Y et al 2006 Sol. Energy Mater. Sol. Cells 901773

[20] Ayyanar M and Babu P S 2012 Asian Pac J. Trop. Biomed. 2 240

[21] Manoharan D, Loganathan A, Karapati V and Nesamony V J 2015 Ultrason. Sonochem. 23174 